\title{
Induction of Supraventricular Tachycardia by Ventricular Pacing: What is the Mechanism?
}

\author{
FRED MORADY, M.D. \\ From the Division of Cardiology, Department of Internal Medicine, University of Michigan, Ann Arbor, Michigan
}

\section{Case Presentation}

A 29-year-old woman underwent an electrophysiologic procedure because of a 10-year history of recurrent episodes of paroxysmal supraventricular tachycardia. The baseline cycle length, atrial-His (AH), and His-ventricular (HV) intervals were 550,50 , and $45 \mathrm{msec}$, respectively. The AV block cycle length was $270 \mathrm{msec}$. With programmed atrial stimulation, there was no evidence of dual AV nodal pathways or inducible tachycardia. During ventricular pacing, there was occasional VA conduction at cycle lengths of 500 to $400 \mathrm{msec}$, and VA dissociation at paced cycle lengths below 400 msec (Fig. 1). On several occasions upon cessation of ventricular pacing, a supraventricular tachycardia with a cycle length of $300 \mathrm{msec}$ was initiated (Fig. 2). What is the mechanism of the tachycardia, and how was it initiated?

\section{Commentary}

The supraventricular tachycardia has a long RP interval, which is consistent with orthodromic AV reentrant tachycardia, atrial tachycardia, or atypical AV nodal reentrant tachycardia. Because AV reentrant tachycardia requires an accessory pathway capable of retrograde conduction, the absence of VA conduction when pacing the ventricle at cycle lengths equal to or longer than the tachycardia cycle length rules out this mechanism of tachycardia. Furthermore, in the absence of VA conduction, there would be no direct mechanism by which ventricular pacing could induce an atrial tachycardia. Therefore, by exclusion, atypical AV nodal reentry is the most likely mechanism of the tachycardia.

To induce AV nodal reentry in the absence of VA conduction, ventricular pacing must have had an effect on the AV node; however, events that occur within the AV node cannot be recorded with conventional electrode catheters in the clinical electrophysiology laboratory. For this reason, the mechanism by which ventricular pacing resulted in induction of atypical AV nodal reentrant tachycardia must be inferred by deductive reasoning.

A possible mechanism arrived at by deductive reasoning

This manuscript was processed by a guest editor.

J Cardiovasc Electrophysiol, Vol. 13, pp. 1306-1308, December 2002.

Address for correspondence: Fred Morady, M.D., Division of Cardiology, Department of Internal Medicine, University of Michigan, 1500 E. Medical Center Drive, B1F245, Ann Arbor, MI 48109-0022. Fax: 734-936-7026; E-mail: fmorady@umich.edu is shown in Figure 3. A sinus beat penetrates both the fast and slow AV nodal pathways, and conducts to the His bundle through the fast pathway. The wavefront that exits the fast pathway enters the distal slow pathway, causing a collision within the distal portion of the slow pathway. A wavefront resulting from a paced ventricular complex then conducts to the AV node and retrogradely penetrates the fast and slow pathways, but blocks in both pathways because the proximal portions are refractory. However, because the effective refractory period of the fast pathway is longer than that of the slow pathway, the wavefront penetrates the slow pathway to a greater degree than the fast pathway.

The next sinus beat again penetrates both the fast and slow pathways, but this time, because of the concealed retrograde penetration from the preceding paced complex, the wavefront blocks in a proximal portion of the slow pathway. After anterograde conduction through the fast pathway, the wavefront enters the slow pathway and finds it excitable. Because the proximal portion of the slow pathway has regained its excitability by the time the retrograde wavefront has advanced to the proximal slow pathway, the wavefront is able to conduct through the entire slow pathway, activate the atrium, and reenter the fast pathway, thereby initiating the atypical AV nodal reentrant tachycardia.

How can a patient who has atypical AV nodal reentrant tachycardia at a cycle length of $300 \mathrm{msec}$ have VA dissociation when ventricular pacing is performed at longer cycle lengths in the setting of sinus rhythm? This often may be attributable to VA block in the common distal pathway, below the reentrant circuit. However, in the present case, if block had occurred in the common distal pathway during ventricular pacing, the wavefront would not have been able to penetrate the fast or slow pathway, or result in the initiation of tachycardia. Therefore, it is more likely that VA block was caused by concealed penetration of the fast and slow pathways by sinus beats, resulting in retrograde block in these pathways, as shown in Figure 3. At slower pacing rates, there would be more time for the fast and slow pathways to recover excitability after concealed penetration by a sinus beat, explaining why there was occasional VA conduction at ventricular paced cycle lengths $\geq 400 \mathrm{msec}$.

Another possible explanation for the induction of the tachycardia might be that ventricular pacing evoked an atrial premature depolarization that then induced the tachycardia; however, this explanation is not logical for two reasons. First, in the absence of VA conduction, it is unlikely that ventricular pacing would induce an atrial premature depolarization. Second, as shown in Figure 2, the first 


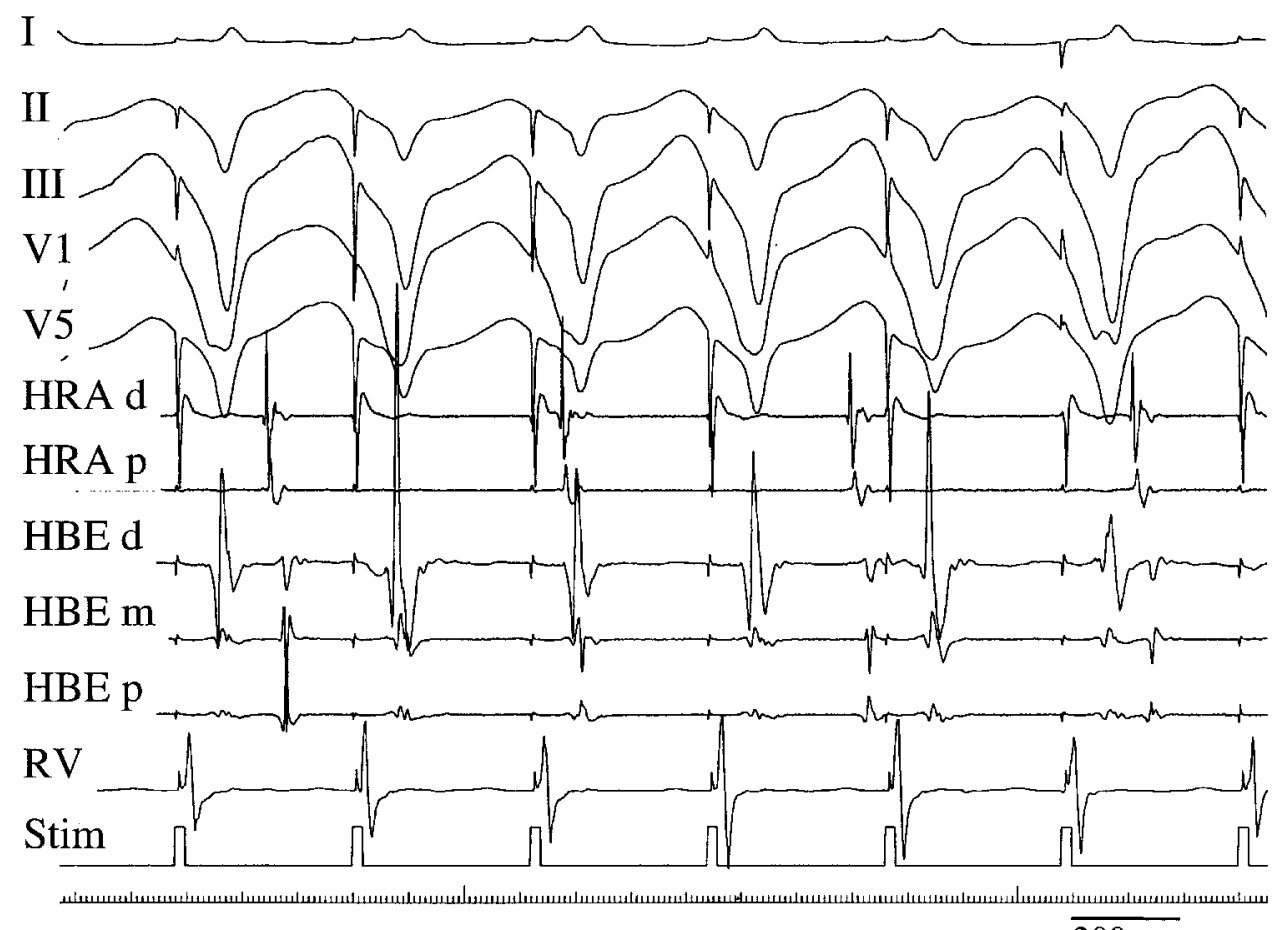

Figure 1. Ventriculoatrial dissociation during ventricular pacing at a cycle length of $320 \mathrm{~ms}$. Shown are recordings from leads $I$, II, III, $V_{1}$, and $V_{5}$, high right atrial (HRA), His bundle (HBE), and right ventricular (RV) electrograms, and a stimulus (Stim) channel. $d=$ distal; $m=$ mid; $p=$ proximal.

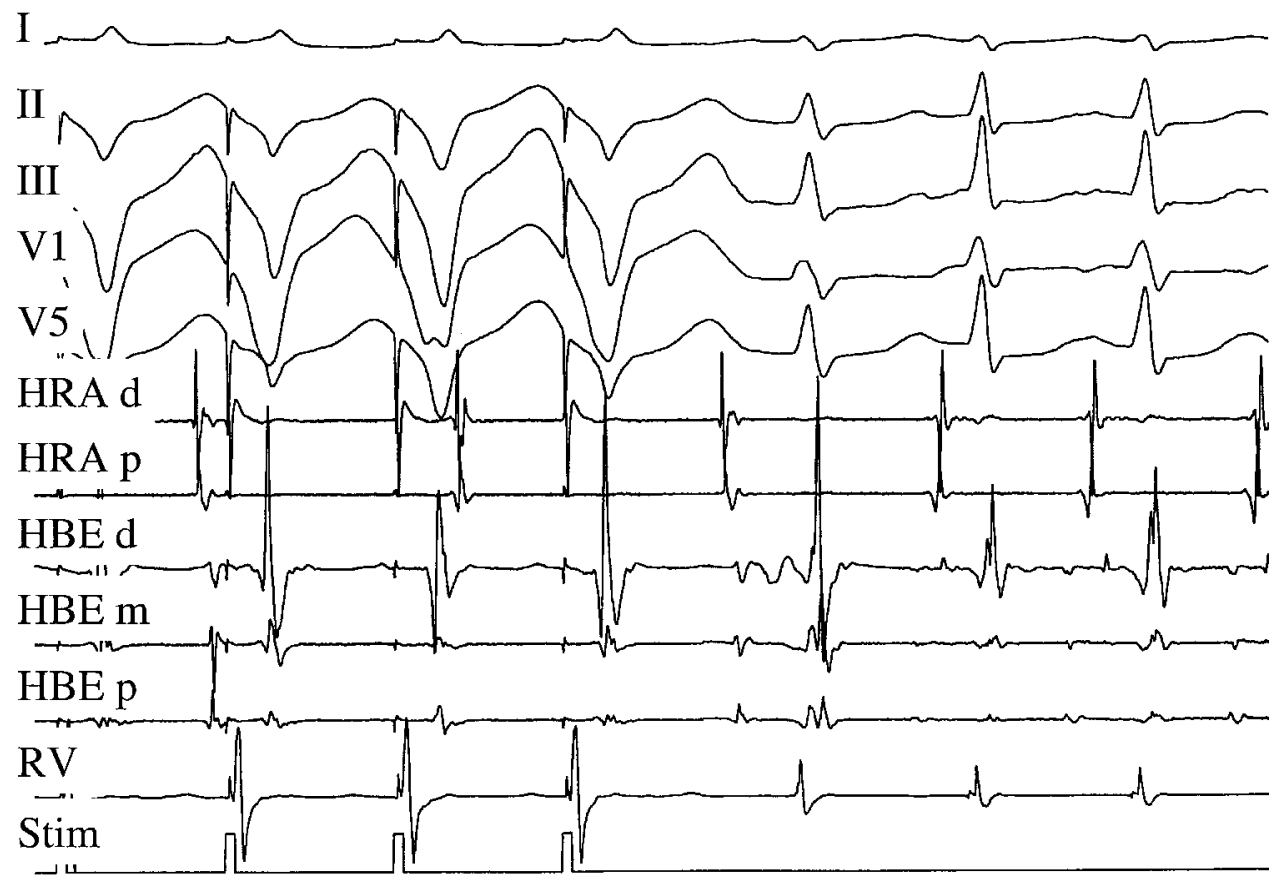

$200 \mathrm{~ms}$

Figure 2. Upon cessation of ventricular pacing at a cycle length of $320 \mathrm{~ms}$, a sinus beat is followed by the onset of tachycardia that has a cycle length of 300 to 310 ms. Format and abbreviations as in Figure 1. 


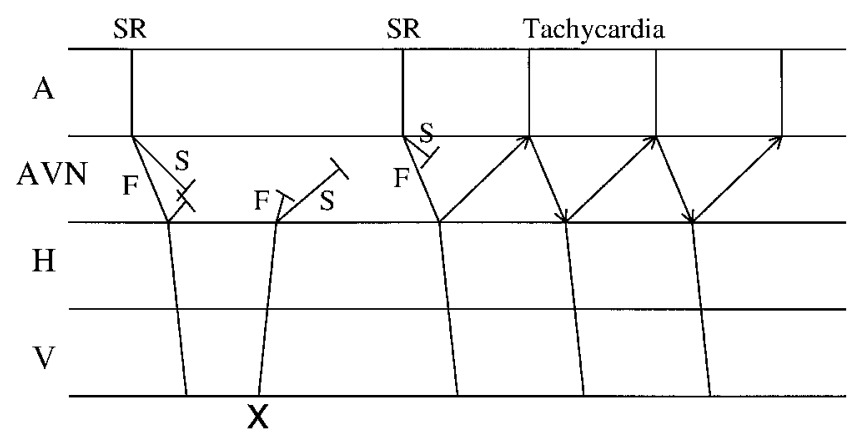

Figure 3. Proposed explanation for induction of atypical AV nodal reentrant tachycardia after ventricular pacing. See text for explanation. $X$ denotes a ventricular paced complex. $A=$ atrium; $A V N=$ atrioventricular node; $F=$ fast pathway; $H=$ His bundle; $S=$ slow pathway; $S R=$ sinus rhythm; $V=$ ventricle. complex after the cessation of ventricular pacing is a sinus beat, and it is the next beat that might be considered a premature atrial depolarization that induces tachycardia. Even if ventricular pacing had induced an atrial premature depolarization in the absence of VA conduction, there would be no way to explain how a sinus beat could occur between the last ventricular paced complex and the premature depolarization.

Although the mechanism proposed in Figure 3 cannot be proven, at least it is logical and consistent with basic electrophysiologic concepts. Sometimes this is the strongest argument that can be made to support an explanation for events that are concealed within the AV node.

Radiofrequency slow pathway ablation in the posteroseptal right atrium, near the coronary sinus ostium, was effective in eliminating the tachycardia. 